تصميم الثكل الهندسي للاراع الحديدي في العدسة الكهرومغناطيسية المكثفة-الثيئية

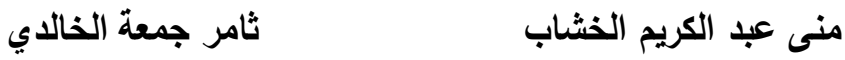 \\ قسم الفيزياء/ كلية العلوم / جامعة الموصل
}

(أستلم 2013/24 ؛ قُبل 20 20 / 1 / 2014)

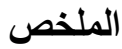

إن تحسين تركيب القطب الرئيسي والثكل الهندسي للذراع الحديدي في العدسة المغناطيسية ثنائية القطب اللامتتاظرة والتي تعمل بوصفها عدسة مكثفة-شيئية أدى إلى أمكانية تصميم عدسات المجهر الالكتروني بقدرات تحليل عالية جدا.

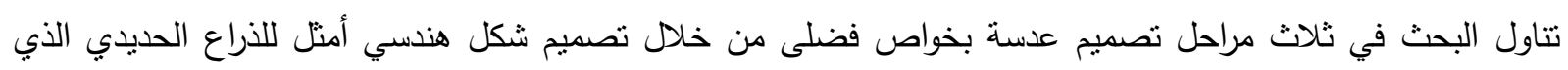
يحقق أعلى ذروة فيض للمجال المغناطيسي وأقل معامل زيغ كروي عندما تعمل العدسة بفولنية النتخغيل المصححة نسبياً وتهيج ثابت (VI=200 kV)

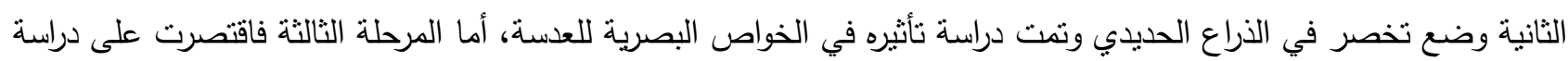
تأثير بعد موقع التخصر عن المحور البصري على أداء العدسة. ونم احتساب نوزيع كثافة المجال المغناطيسي، ومسارات خطوط الفيض المغناطيسي في تركيب العدسة، وخواصها البؤرية للمراحل الثناث لتتخيص العيوب وتحسين الثنكل الهندسي للذراع الحديدي والأداء البصري، وتم اختيار القيم المنلى للخواص البؤرية الثيئية للعدسة المقترحة. ووجد ان معامل البعد البؤري ينتاسب

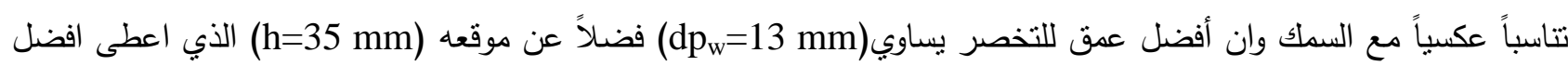
قدرة تحليل للعدسة (S) وقد تمت مقارنة النتائج للبحث الحالي مع نظيراتها من البحوث المنشورة ووجد أنها تتفق تماما.
\end{abstract}

الكلمات الدالة: العدسة الكهرومغناطيسية المكثفة-الثيئية، قدرة تحليل العدسة الكهرومغناطيسية، تصميم العدسة الكهرومغناطيسية ثنائية القطب اللامتناظرة.

\title{
The Design of the Iron Shroud Geometrical Shape in the Condenser- Objective Electromagnetic Lens
}

\author{
Muna A. Al-Khashab \\ Thamer J. Al-Khaldey \\ Department of Physics / College of Science/ University of Mosul
}

\begin{abstract}
The optimization of the main polepiece structure and the geometrical shape of iron shroud in the asymmetric double polepiece magnetic lens for the condenser-objective mode led to the possibility of the higher resolution lens design for the electron microscope.

This work is handled by three steps, the designed lens having optimum properties through the construction of a fine geometrical shape of the iron shroud which results the highest flux density peak of the magnetic field with a lowest spherical aberration coefficient, at $\left(\mathrm{V}_{\mathrm{r}}=200 \mathrm{kV}\right)$ and excitation of $(\mathrm{NI}=10.5 \mathrm{kA}-\mathrm{t})$. In the first step the thickness of the iron shroud was studied whereas the optical properties of the lens were studied, by putting west point in the iron shroud in the second step. Moreover, the effect of the height of the west point position on the optical performance was
\end{abstract}




$$
\text { منى عبد الكريم الخشاب و ثامر جمعة الخالدي }
$$

studied in the third step. The Calculation has been made for the axial magnetic field distribution, the magnetic flux lines trajectories and the optical properties to limit the defects in the lens and consequently improve the geometrical shape of the iron shroud. It was found that the focal length inversely proportional to the thickness of the iron shroud and the best depth for the west point equals $\left(\mathrm{dp}_{\mathrm{w}}=13 \mathrm{~mm}\right)$, beside that the west point position was $(\mathrm{h}=35 \mathrm{~mm})$ which gave the best resolving power $(\delta=0.2 \mathrm{~nm})$.

The obtained results of the preferred properties in the present work have been compared with those of the published results, and it was found that both results are in a good agreement with each other.

Keywords: The condenser-objective electromagnetic lens, the higher resolution electromagnetic lens, asymmetric double polepiece electromagnetic lens design.

\section{المقدمة}

Al-Khashab and ) إن أفضل تصميم للعدسة الكهرومغناطيسية ذات معاملات الزيوغ الواطئة يعتبر ذو أهية كبيرة (Ahmed, 2011 يمكن من خلال تغيير الثكل الهندسي اللعدسات للحصول على أفضل الخواص البصرية. إذ أن العدسة الكهرومغناطيسية التي تمنلك أعلى ذروة فيض بأقل عرض نصفي للمجال المغناطيسي نتتج زيوغاً واطئة (Mulvey, 1982). وقد حاول الباحثون

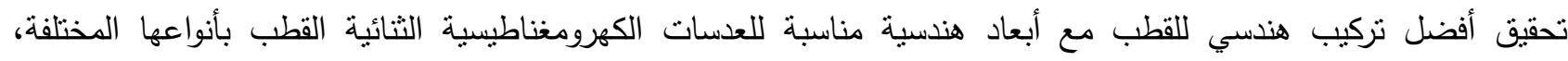
المتتاظرة التي قام بها (Cleaver, 1980) واللامتتاظرة التي أجراها (Wenxiong, 1988). وتمت دراسة العدسات الأحادية القطب اللامتتاظرة من قِبل (Al-Khashab and Abbas, 1991) لنفس الهدف. ومع تطور تقنيات النانو أصبح من المهم تحسين قدرة التحليل (Resolving power) لعدسات المجهر الالكتروني وفق المعايير النانوية، ويتم ذلك بتقليل الزيوغ إلى أدنى حد ممكن في العدسة الثيئية (Li et al., 2009)، وتُبئَ العدسة الككثقةالثنيئية بطريقة (Riecke) لإثباع القطب بالفيض المغناطيسي وتوليد أعلى ذروة كثافة فيض مغناطيسي في مركز العدسة (Mulvey , 1986)، إذ تختلف العدسة المكثفة-الثيئية عن العدسة الثيئية التقليدية بوضع النموذج في منتصف الفجوة الهوائية حيث تعترض الجهة الأمامية العليا للنموذج المجال الأمامي (Pre-field) وتعمل كعدسة مكثقة، أما الجهة الخلفية السفلى (S) للنموذج فتعترض الجزء الخلفي للمجال (Post-field) المار في العدسة وتعمل كعدسة شيئية (2005, Ray). أي أن مركز العدسة يكون منتصف الفجوة الهوائية وبذلك فأن الجزء الواقع أمام النموذج يعمل كمنظومة إضاءة، والجزء الواقع خلف النموذج يعمل كمنظومة تصوير (Tsuno and Harada, 1983)، وللعدسة المكثقة-الثنيئية أهمية كبيرة في كلا المنظومتين سواءً الإضاءة منها أو التصوير (Krivanek et al., 2008). حيث يتعرض النموذج إلى مجال عالي جداً في العدسة الككثة-

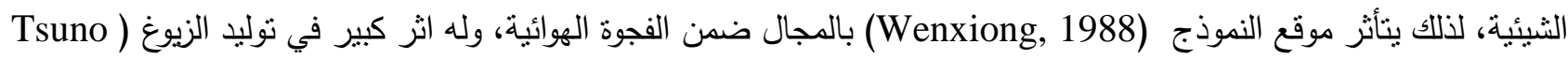
Electron probe )

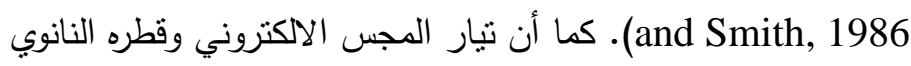
(1986) يعد من أهم العوامل في دراسة الخواص البصرية للجانب الأمامي (المكثف) للعدسة المكثفة-الثنيئية (diameter 1 nm Yanaka,

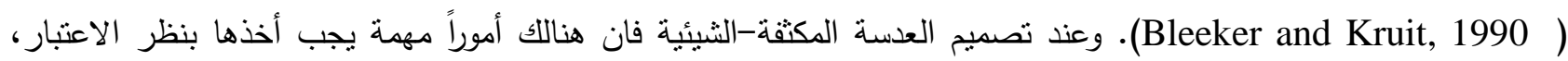
كشكل وحجم الملفات والقدرة على تبريدها (Mulvey, 1982) بالإضافة إلى الحفاظ على استقرارية مقدار التهيج العالي فيها، أعلى من واحد أمبير لكل مليمتر مربع (Podbrdsky, 1986). 
يتضمن البحث الحالي تصميم شكل هندسي للذراع الحديدي للعدسة المكثقة-الثنيئية ودراسة خواصها البؤرية الثييئة للحصول على أفضل شكل هندسي للذراع الحديدي بحيث يحقق اقل زيوغ وأعلى قدرة تحليل ممكنة للعدسة في حالة نتغيلها بفولتية تعجيل مصححة نسبياً مقدارها) وتهيج ثابت يساوي ( V V .(Infinite magnification condition )

\section{تصميم العدسة الكهرومغناطيسية المكثفة-الثيئية ثنائية القطب اللامتناظرة}

إن تصميم العدسات الكهرومغناطيسية يتطلب تحديد أفضل تركيب هندسي للقطب وشروط التشغيل المرافقة لها (فولنية

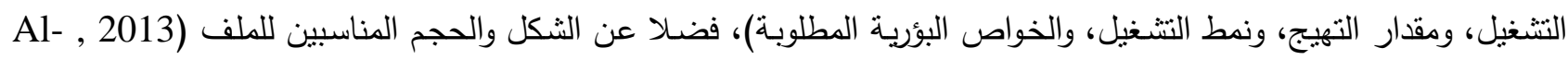
.(Khashab and Al-Hialey

Finite (FEM) إن التقنية المميزة والمستخدمة في تصميم العدسات الكهرومغناطيسية هي طريقة العناصر المتتاهية Element Method (Lencova' , 1986) (Meshes) ) إضافة إلى طرائق الحساب النظري والتحليل العددي.

لقد عُدِد في البحث الجاري إلى تصميم عدسات ثثائية القطب لا متتاظرة والتي تنتشابه في جميع معلماتها وأبعادها وأثنكالها الهندسية باستثناء الذراع الحديدي الذي يتغير شكله الهندسي مع تغيير العدسة المقترحة لتعمل عند فولنية التشغيل المصححة نسبياً (التهيج الثابت (NI=10.5 kA-t) بنمط العدسة المكثة-الشيئية، التي لا تحتاج إلى قيم تهيج عالية جدا. حيث أن شكل القطب الأول (First polepiece) يكون مخروطياً ناقصاً، ارتفاع وجه القطب فيه (b) (b) وزاوية ارتفاعه

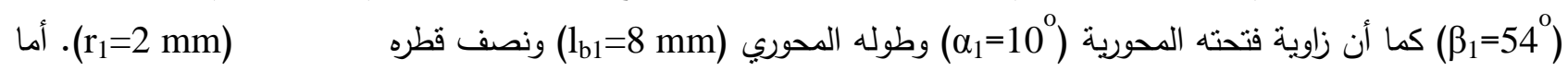

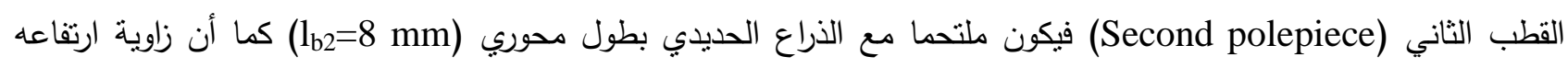
يساوي(

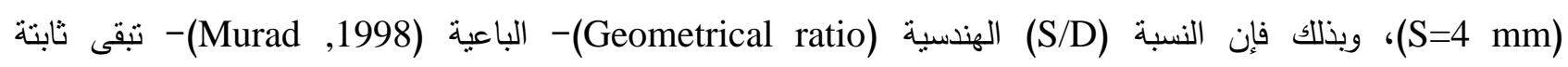
وهي قيمة منوافقة مع المعايير المحدة في تصميم العدسات الثنائية القطب (S/D=0.89)

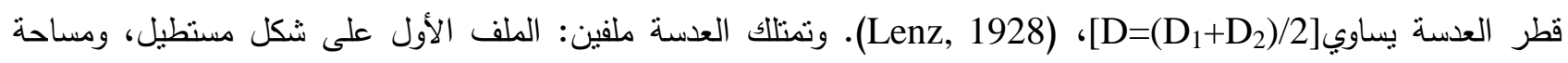
مقطعه العرضي $\left(\mathrm{A}_{1}=500\right.$ mm². والملف الثاني ذو شكل رباعي أضلاعه غير منساوية بالطول تتقاطع بزوايا مختلفة،

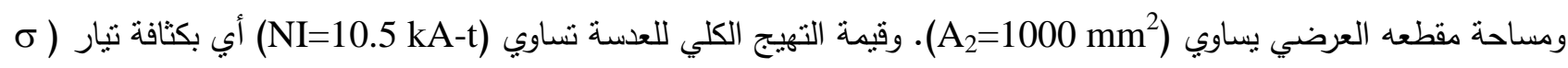

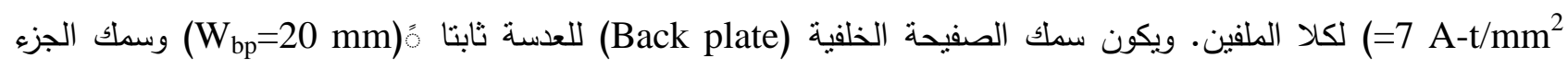

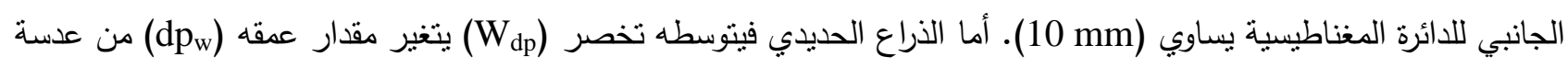
إلى أخرى حسب التصميم المطلوب، كما هو موضح في الثكل (1). 


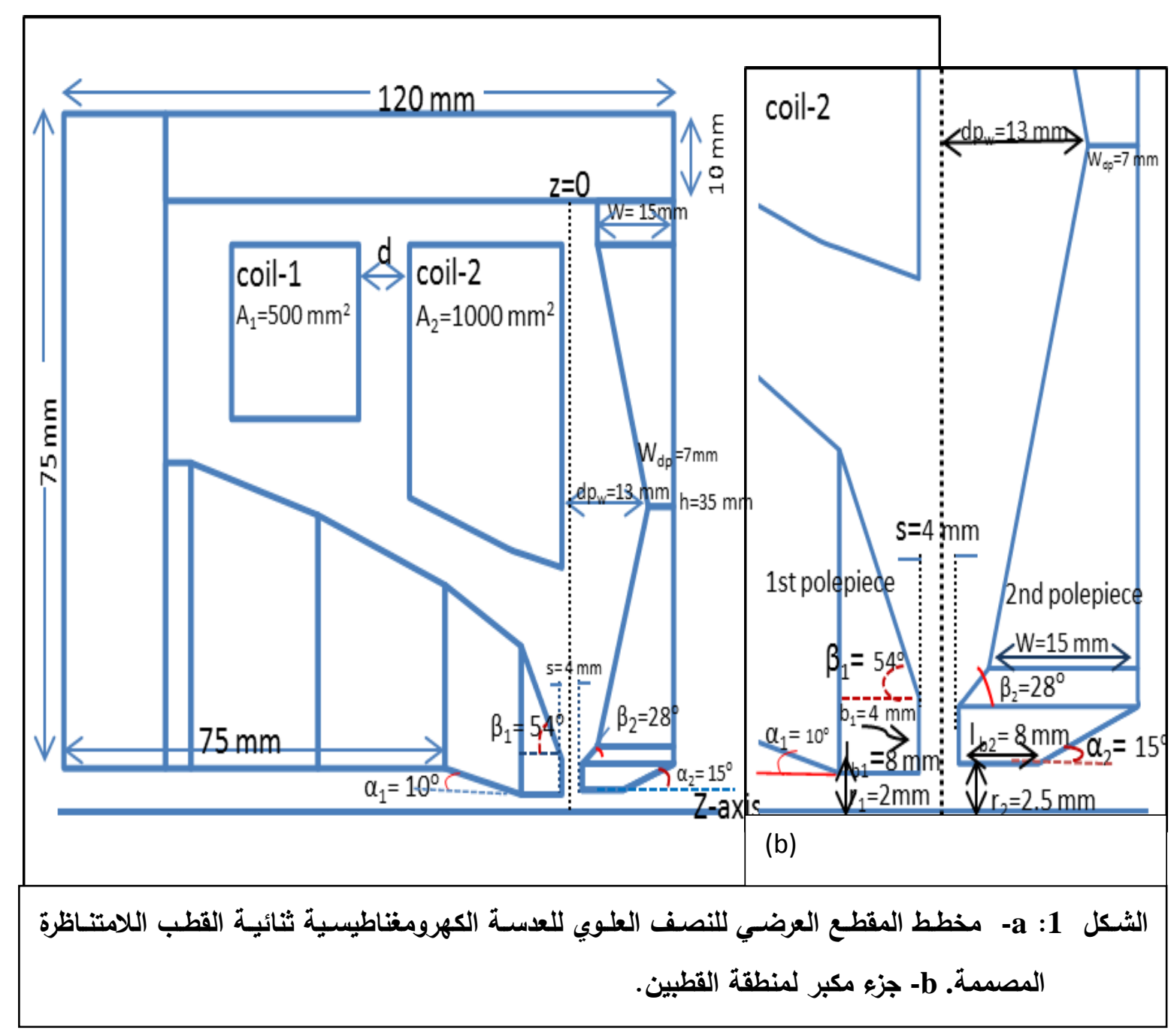

يتألف البحث من جزئين: الجزء الأول يختص بدراسة تغيير سمك الذراع الحديدي وأهمية تأثيره في الخواص البؤرية

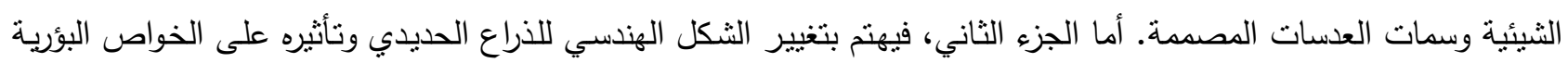

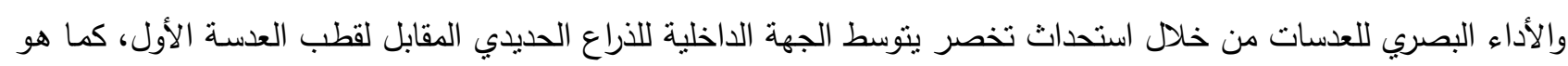
مبين في الثكل(1) أعلاه. وهذا المقترح سيتم استقصاؤه ضمن هذا البحث لأول مرة في مجال البصريات الالكترونية. فقد تمت

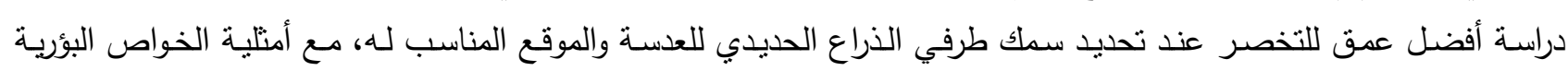
المترافقة عند فولتية التتغيل المطلوبة (VI=200 kV) للعدسة المكثفة-الثنيئية عند شرط التكبير اللامحدود. وتمت معايرة جميع النتائج عند هذه الفولتية باستخدام المعادلة التالية:

$\mathrm{n}=\left(\mathrm{V}_{\mathrm{r}} / \mathrm{V}_{\text {original }}\right)^{1 / 2}$

حيث أن Vوr فولتية التعجيل المصححة نسبياً (فولتية التتغيل) المطلوبة لمعايرة الخواص البصرية عندها وتساوي (200 kV). و (Voriginal) الفولتية النطبيقية (الأصلية) المصححة نسبياً التي تعطي الخواص البؤرية وتكون عندها كثافة المجال المغناطيسي بأعلى ذروتها في مركز العدسة (Z=0.0) منتصف الفجوة الهوائية. و n يمثل معامل المعايرة. 
الجزء الأول: تأثير سمك الذراع الحديدي(W) على الخواص البؤرية الثيئية للعدسات المكثفة-الشيئية نم في هذا الجزء من البحث اختبار ثلاث قيم مختلفة لسمك الذراع الحديدي (W) وهي (W)

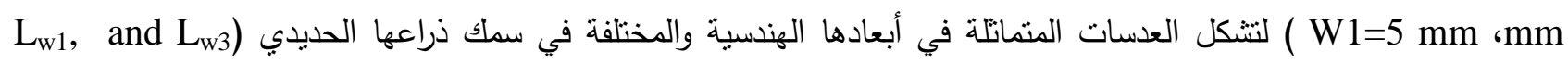

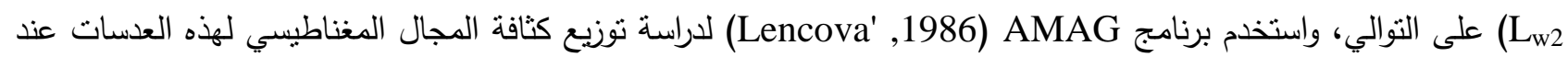

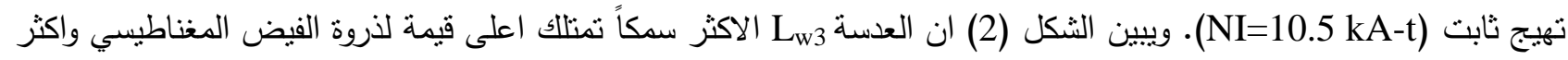
انتظاماً للمجال من بقية العدسات ونتوسط الذروة منطقة الفجوة الهوائية فيها. 1998) ونت احتساب مسارات خطوط الفيض المغناطيسي في تركيب العدسات أعلاه باستخدام برنامج

(Murad ,

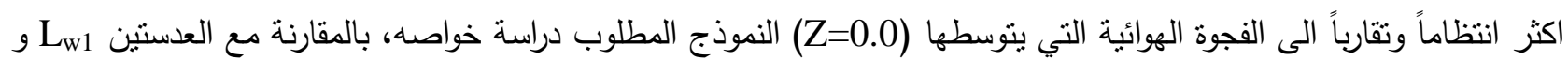

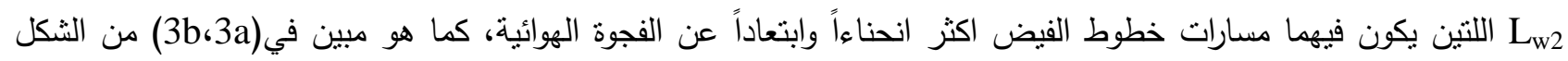
(3). وبشكل عام فان هذه الخطوط نجدها منتظمة ومنوازية لبعضها البعض ونسقط عموديا على سطح القطب المائل وتقترب من وجه بروز القطب لتكون أكثر انتظاما وتقاربا. كذلك نلاحظ أن خطوط الفيض للعدسة ذات السمك الأكبر تقترب أكثر إلى المحور البصري، كما في الثكل (3d) الذي يظهر جزءاً مكبراً لمسارات خطوط الفيض المغناطيسي للعدسات الثلاث.

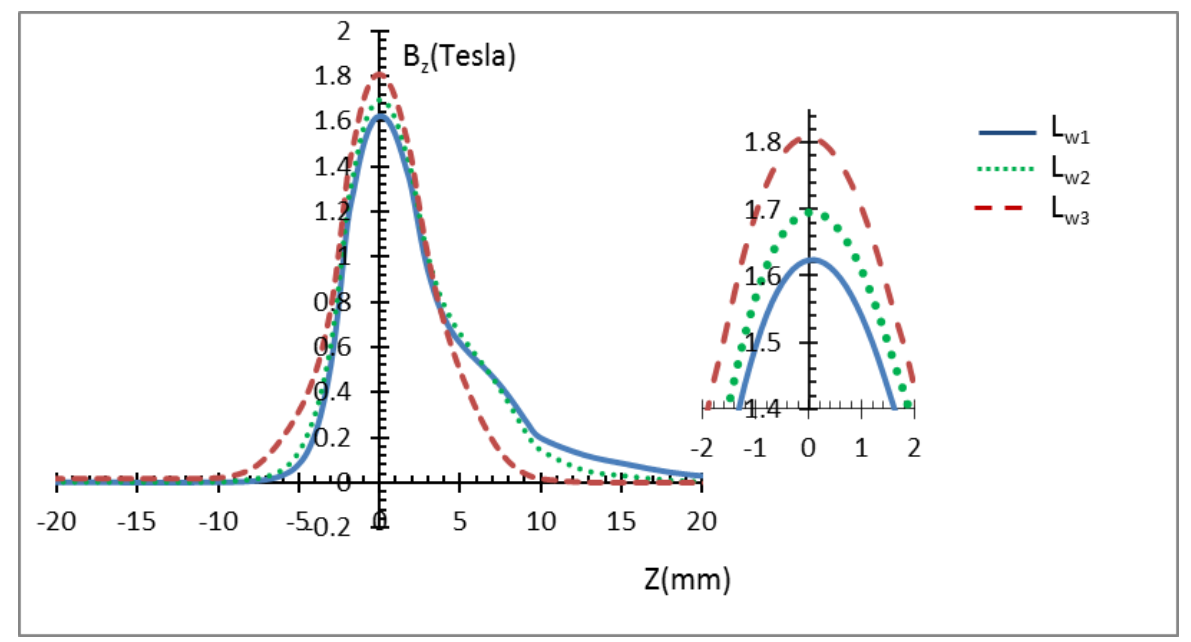

الثكل 2: توزيع كثافة المجال المغناطيسي للعدسات الكهرومغناطيسية الثلاث المختلفة في سـك

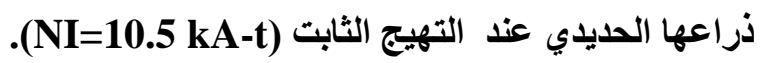

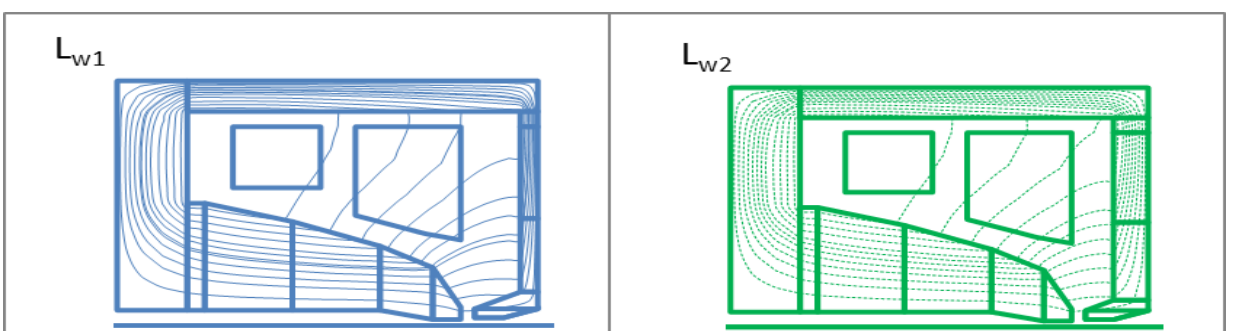


الثكل 3: مسارات خطوط الفيض المغناطيسي في تركيب العدسات الكهرومغناطيسية المختلفة في سمك ذراعها الحديدي عند

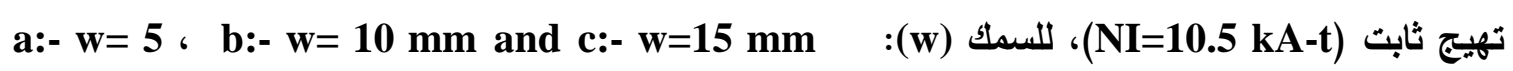

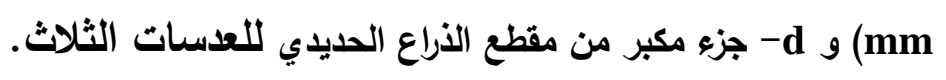

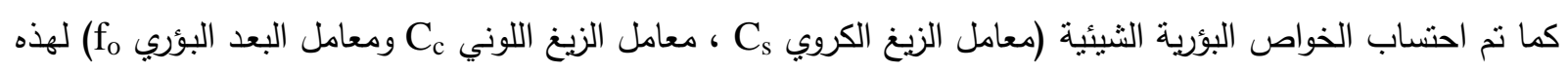

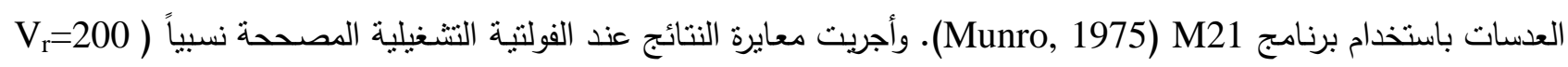

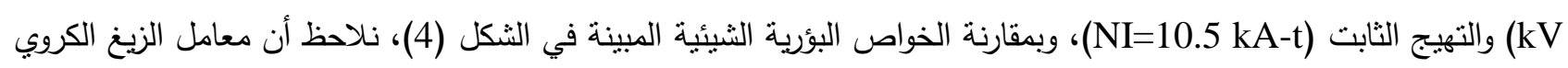
يزداد زيادة نسبية ضئيلة بازدياد سمك الذراع الحديدي، في حين أن معامل الزيغ اللوني يتناقص مع ازدياد التياد سمك الذراع الحديدي، كما ونلاحظ أيضاً أن معامل البعد البؤري بيتاقص بشكل واضح بازدياد سمك الذراع الحديدي، بينما تتحسن قدرة التحليل بتقليل السمك.

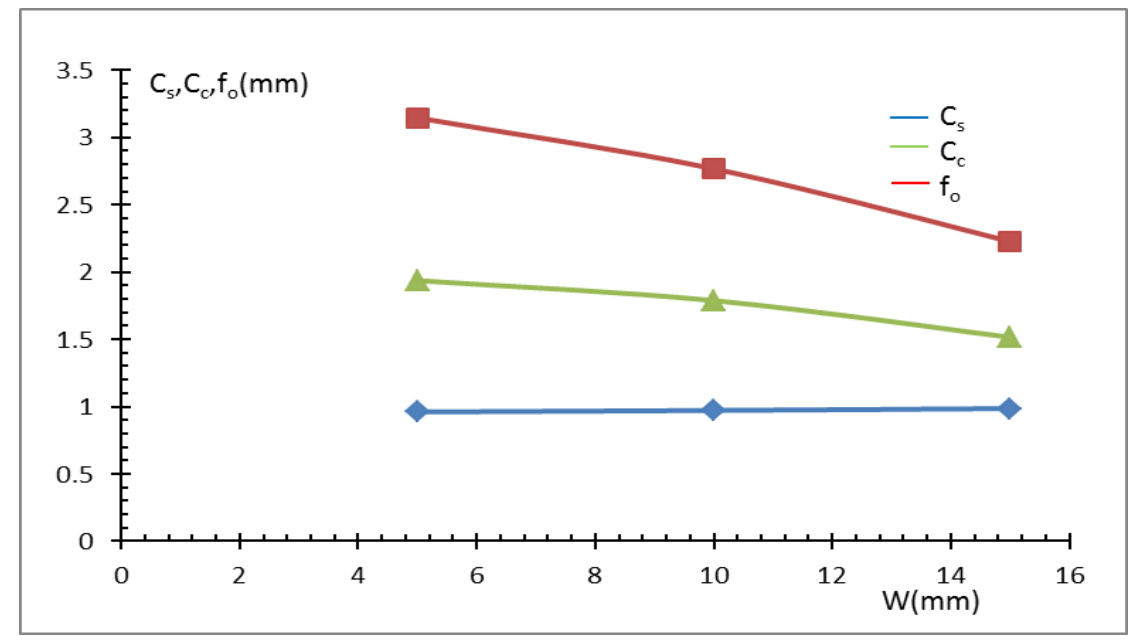

الثكل 4: تغير الخواص البؤرية الثيئية مع تغيير سمك الذراع الحديدي في العدسات الكهرومغناطيسية عند

$$
\text { التهيج الثابت (NI=10.5 kA-t). }
$$


ويبين الجدول رقم (1) مقارنـة الخواص البؤريـة الثيائية فضـا عن كثافة الفيض المغناطيسي لهذه العدسات عند فولتيـة التعجيل المصححة نسبيا (VI=200 kV) بثبوت التهيج (NI=10.5 kA-t) وموقع الجسم عند (Z= (Z) بناءاً على اختبار نمط التشغيل للعدسة (المكثفة -الثشيئية). كما ويوضح الجدول أن القيم النسبية لمعاملي الزيغين الكروي واللوني إلى البعد البؤري

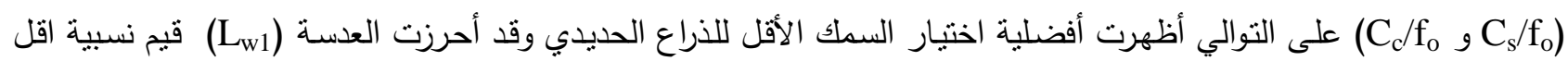

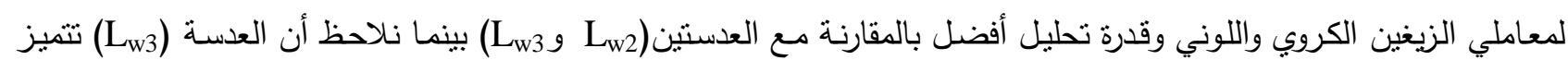

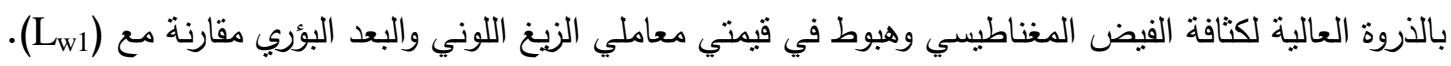

الجدول 1: مقارنة الخواص البؤرية الثيئية للعدسات الكهرومغناطيسية المختلفة في سمك ذراعها الحديدي عند فولتية تعجيل

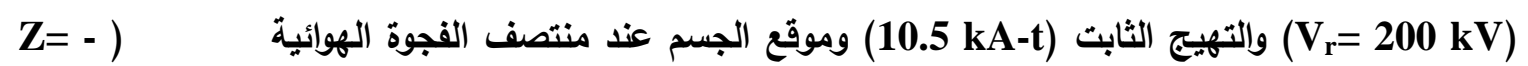

0.04

\begin{tabular}{|l|l|l|l|l|l|l|l|}
\hline $\mathbf{W}(\mathbf{m m})$ & $\mathbf{f}_{\mathbf{o}}(\mathbf{m m})$ & $\mathbf{C}_{\mathbf{s}}(\mathbf{m m})$ & $\mathbf{C}_{\mathbf{c}}(\mathbf{m m})$ & $\mathbf{C}_{\mathbf{s}} / \mathbf{f}_{\mathbf{o}}$ & $\mathbf{C}_{\mathbf{c}} / \mathbf{f}_{\mathbf{o}}$ & $\mathbf{B}_{\mathbf{z}}($ Tesla $)$ & $\boldsymbol{\delta}(\mathbf{n m})$ \\
\hline 5 & 3.146 & 0.960 & 1.937 & 0.305 & 0.615 & 1.344 & 0.266 \\
\hline 10 & 2.767 & 0.971 & 1.788 & 0.351 & 0.646 & 1.384 & 0.266 \\
\hline 15 & 2.227 & 0.985 & 1.515 & 0.442 & 0.680 & 1.650 & 0.267 \\
\hline
\end{tabular}

ومن الجدير بالذكر انه سوف يتم اختيار البعد البؤري (fo=2 mm) بناءاً على الثروط التطبيقية للتشغيل بنمط العدسة

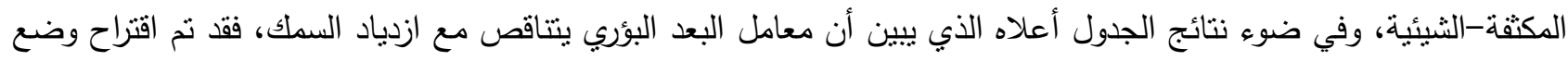
تخصر في الذراع الحديدي اللعدسة يجمع بين السمنين (قلة الزيوغ، وقصر البعد البؤري).

الجزء الثاني: تأثير تخصر الذراع الحديدي(Wdp) على الخواص البؤرية الثيئية للعدسات المكثفة-الثيئية.

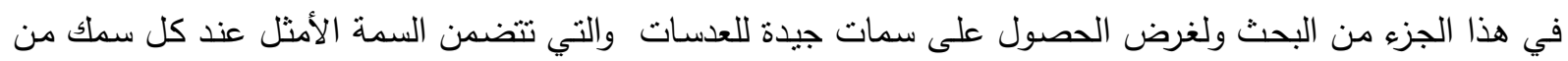
القيم المدروسة في الجزء الأول مجتمعة، فقد تم إحداث تخصر في الجانب الداخلي للذراع الحديدي باتجاه وجه القطب الرئيسي بعمق محدد، وأُفترِضِ أن يكون أفضل موقع له في منتصف الذراع الحديدي بحيث بنتاظر السمك على جانبي التخصر ، فعندما

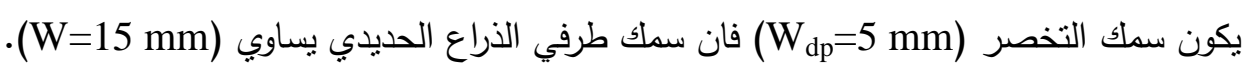

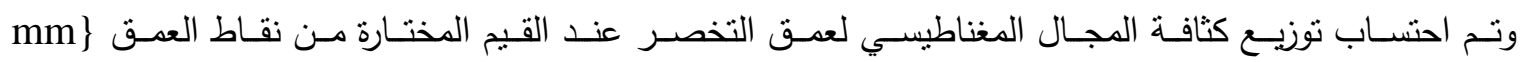
- أي ابتعاد نقطة التخصر عن منتصف الفجوة الهوائية - فوجد أن العمق المناسب للتخصر يكون عند

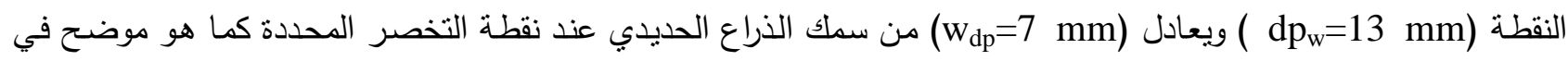
الثكل (5). حيث نلاحظ ارتفاعاً لتوزيع كثافة المجال المغناطيسي عند النقطة (dpw mm ) بينما نجد انخفاضهيا عند النقطة (dpw = 15 mm)

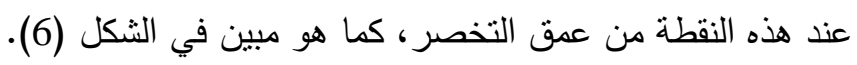

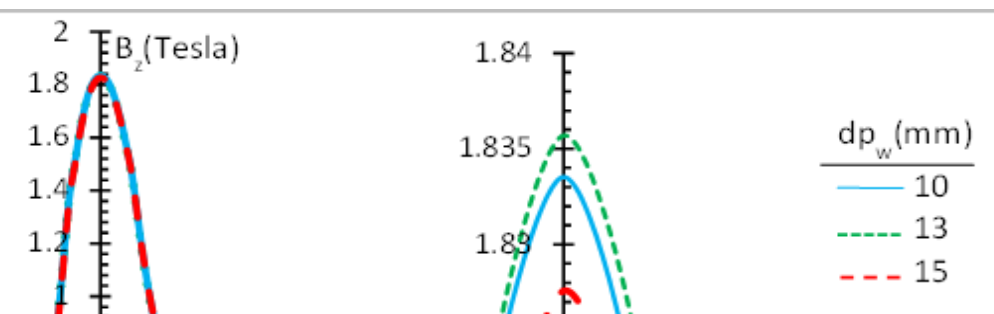


الثكل 5: توزيع كثافة المجال المغناطيسي للعدسات الكهرومغناطيسية مع تغيير عمق التخصر في

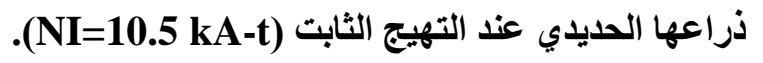

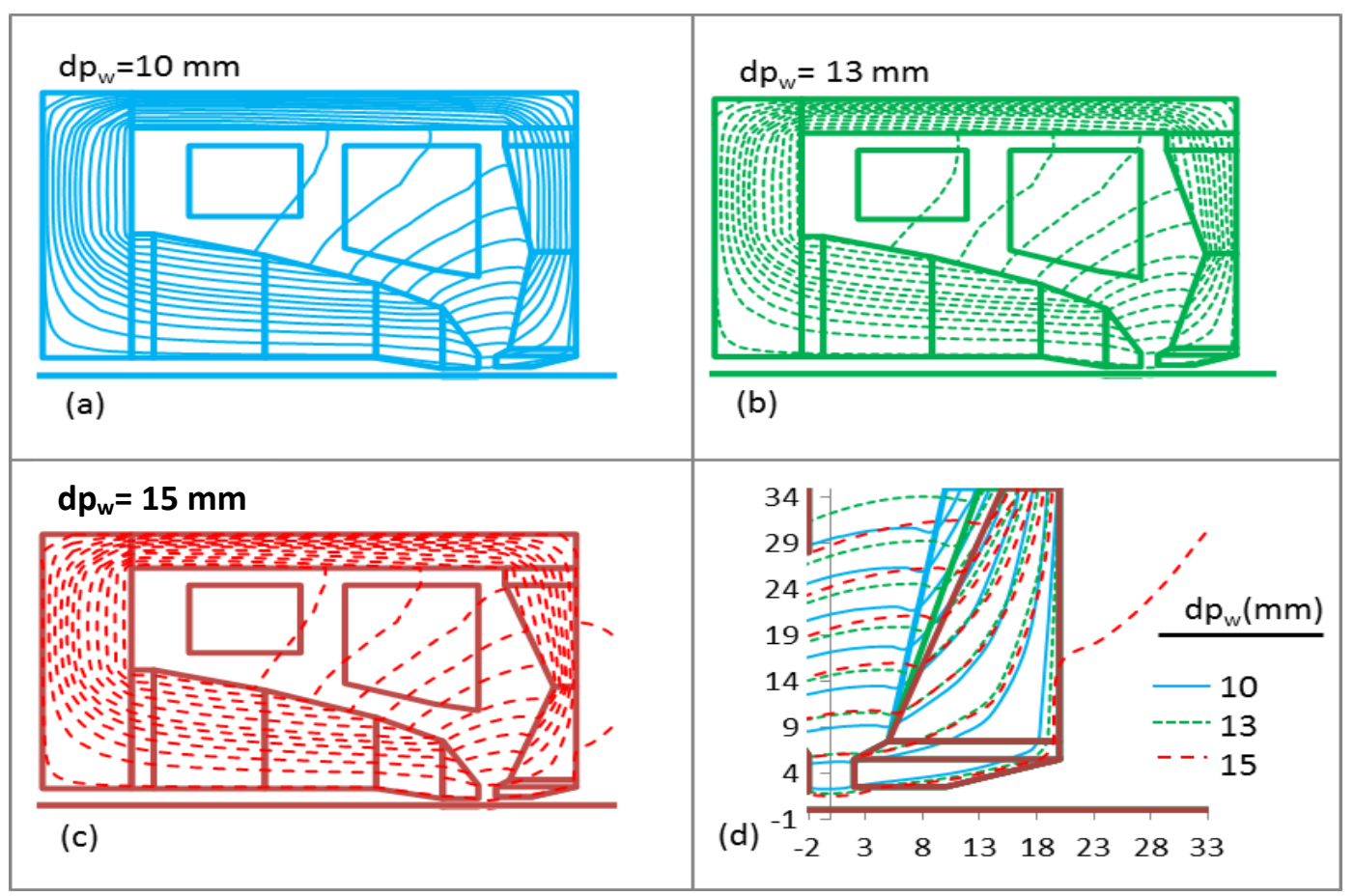

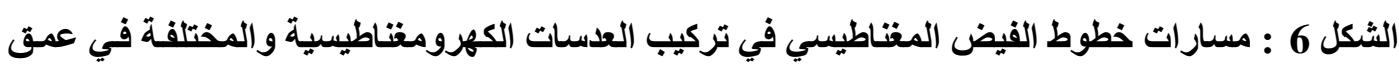

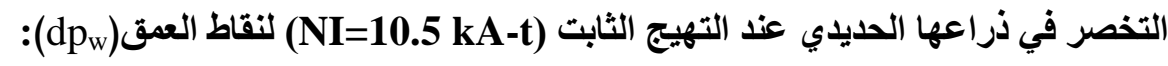

جزء مكبر من مقطع (d ) ( b:- dp 13 mm and c:- dp 15 mm،a:- dp $=10$ mm) الأراع الحديدي للعدسات الثلاث.

وعند احتساب الخواص البؤرية الثيائية لعمق التخصر عند القيم المختلفة من نقاط العمق mm mm (11,12,13،14,15 ، 10)

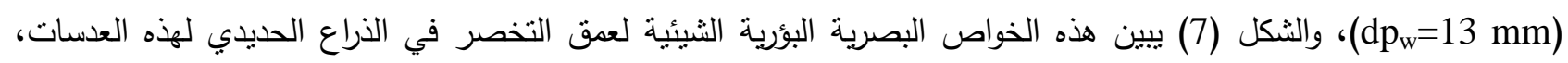
حيث نلاحظ أن أوطئ قيمة لمعامل الزيخ الكروي يكون عند النقطة(dpw mm) كما هو واضح في الشكل (7a)، وان معامل 
الزيغ اللوني ينرافق مع البعد البؤري في الزيادة بازديادعمق التخصر كما في الشكل(7b و 7c

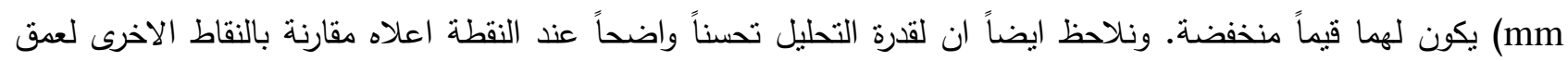
التخصر، كما هو موضح في الثكل (7d).

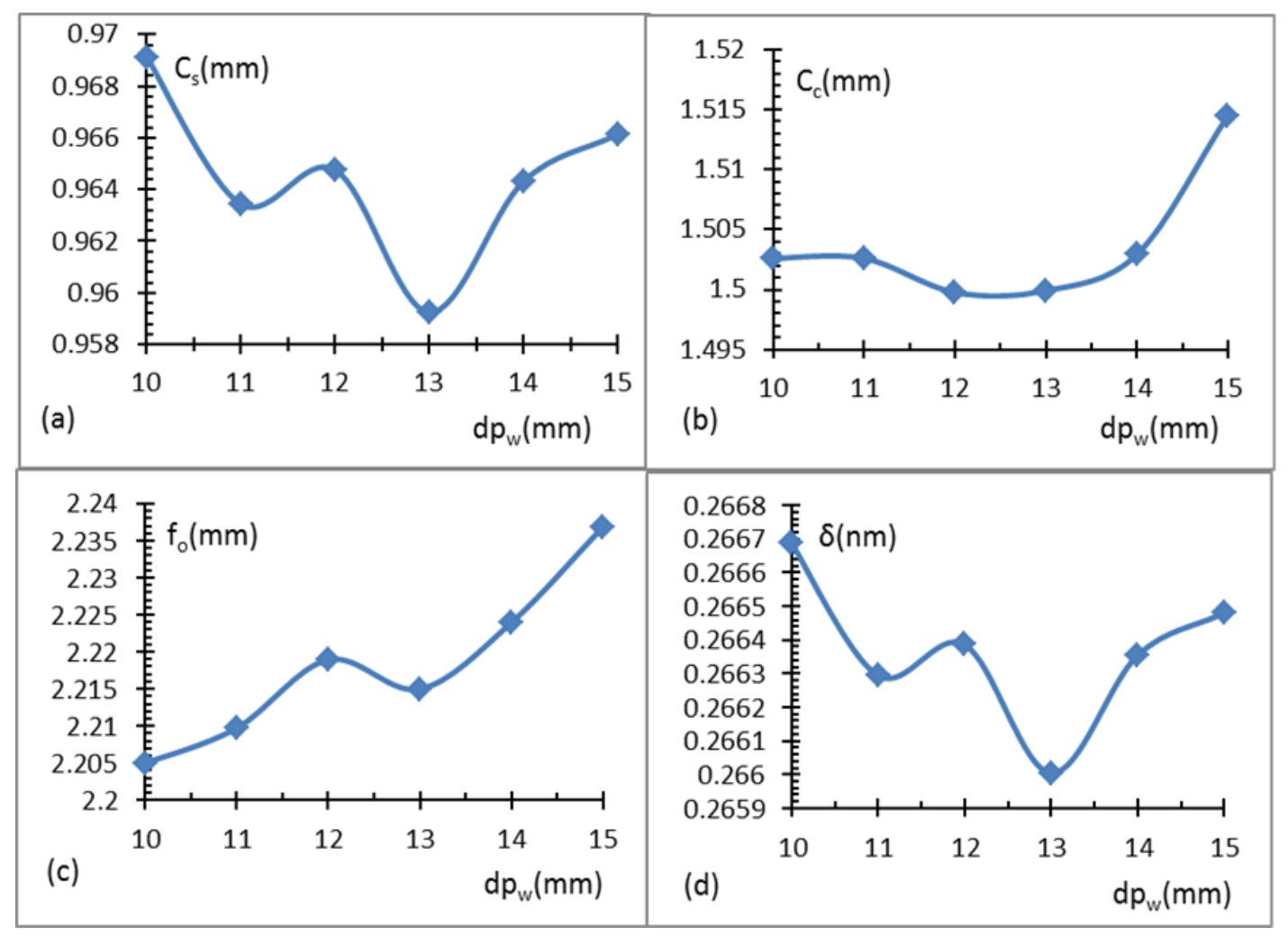

الثكل 7: تغير الخواص البصرية البؤريـة a) ـالزيخ الكروي ، b- الزيغ اللوني، c- البعد البؤري ، d-

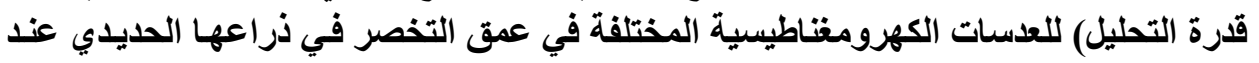

التهيج الثابت (NI=10.5 kA-t).

ويتبين مما سبق ان القيمة الفضلى لعمق التخصر تكون عند النقطة (dpw mm)، لذلك سوف يتم اعتمادها في الخطوات اللاحقة من هذا البحث. حيث سيتم دراسة ازاحة ارتفاع موقع التخصر على استقامة الذراع الحديدي - اي بعده عن

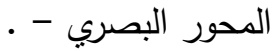

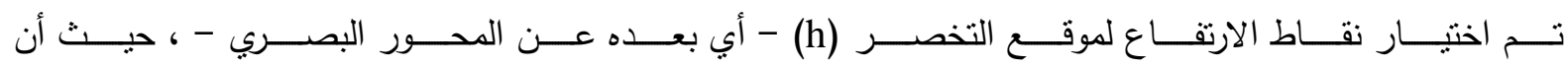

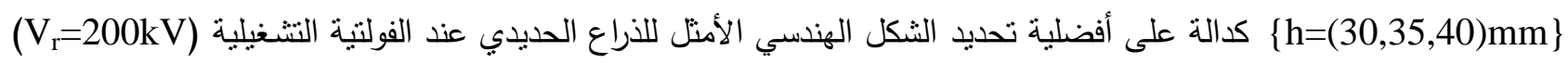
والتهيج الثابت (NI=10.5 kA-t). فلتحديد أمتلية الأداء البصري للعدسات المذكورة أعلاه، فقد تم احتساب نوزيع كثافة المجال

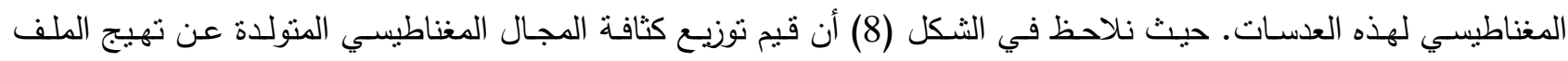

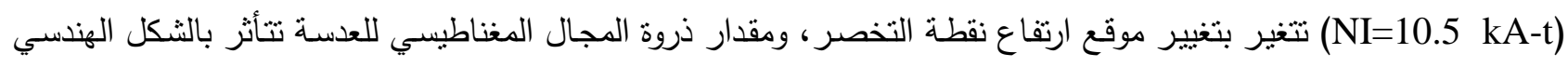

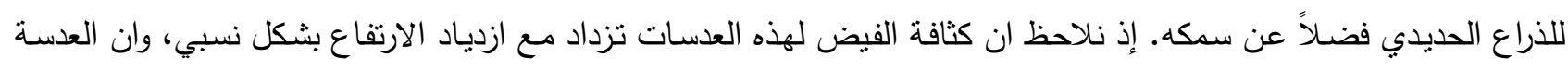
(h35) تتميز بقيمة وسطية لذروة المجال المغناطيسي بثبوت عمق التخصر عند النقطة (dp=13 mm).
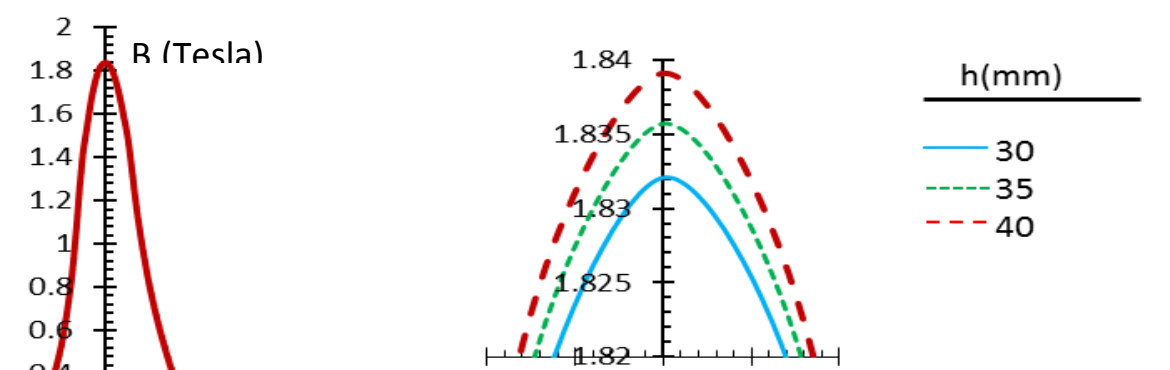
الثكل 8: توزيع كثافة المجال المغناطيسي للعدسات الكهرومغناطيسية بدلالة ارتفاع موقع نقطة التخصر في الأراع الحديدي عند التهيج الثثابت (NI=10.5 kA-t).

كما تم احتساب مسارات خطوط الفيض المغناطيسي في نركيب العدسات أعلاه عند ثبوت التهيج (NI=10.5 kA-t)، ويبين الثكل (9) تغير مسارات خطوط الفيض في تركيب الذراع الحديدي بتغيير ارتفاع موقع التخصر حيث نلاحظ تقارب خطوط

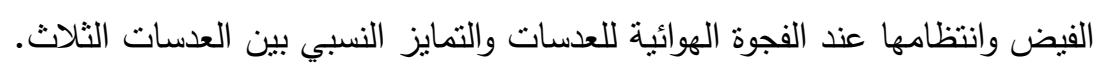

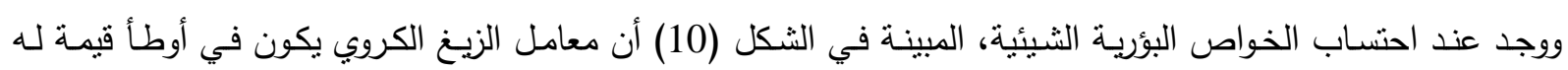
(Cند الارتفاع (h=35 mm) كما هو واضح في الثكل (10a)، في حين أن معاملي الزيغ اللوني والبعد

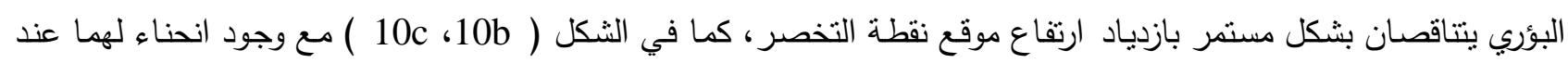
الارتفاع (h=35 mm) لموقع نقطة التخصر في الذراع الحديدي للعدسات المذكورة.

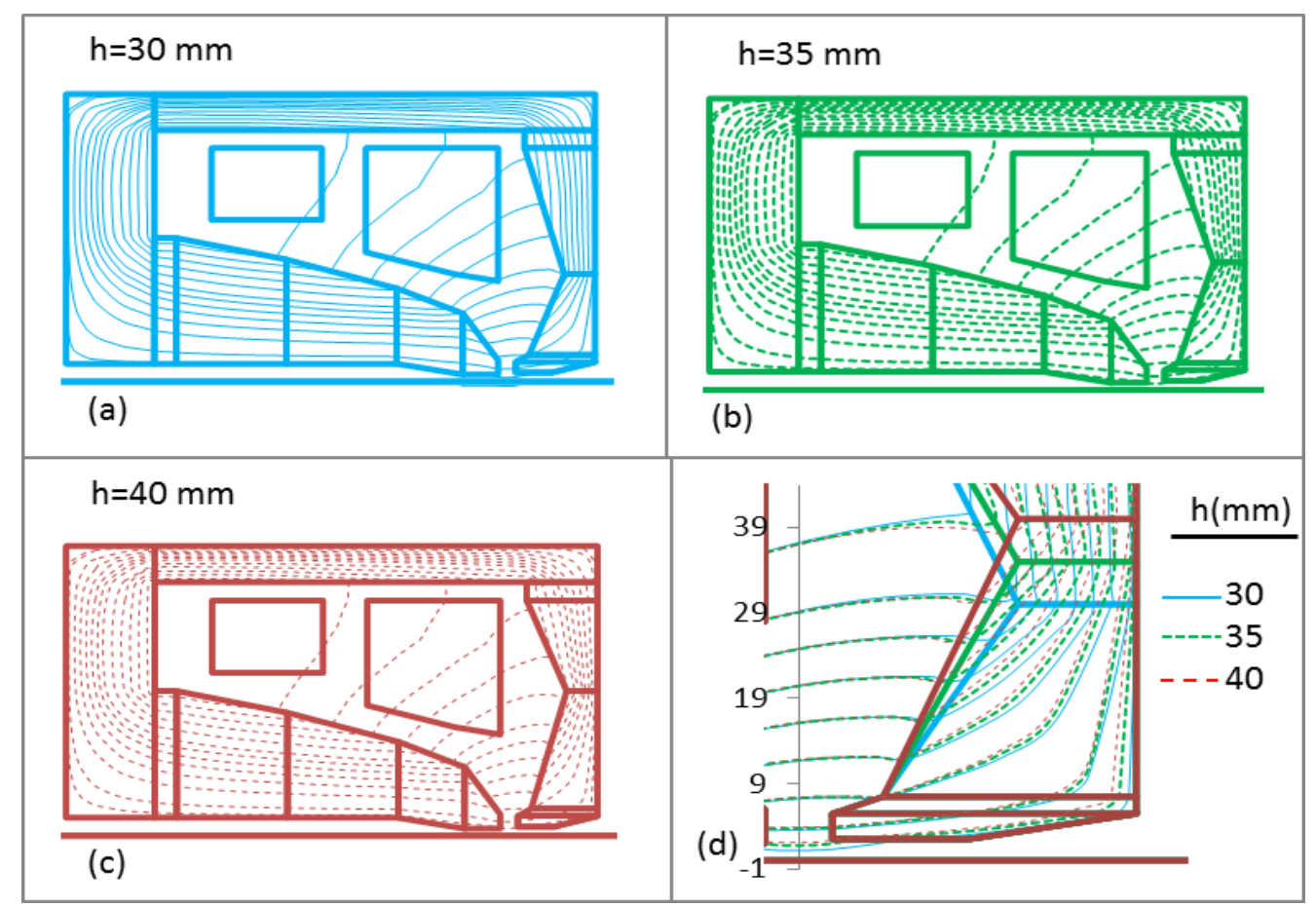

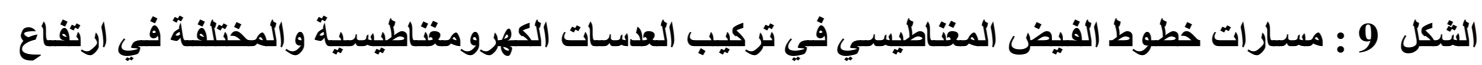

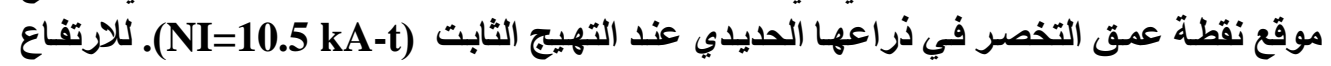
( b:- h= 35 mm and c:- h= 40 mm،a:- h= 30 mm) :(h) الأراع الحديدي للعدسات الثلاث. 
وبتطبيق المعايرة عند فولتية التعجيل المصححة نسبياً (V)=200 kV) والتهيج الثابت (NI=10.5kA-t) وشرط التكبير

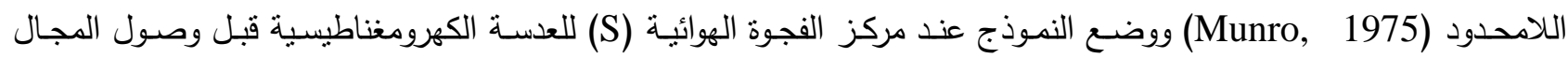
المغناطيسي إلى أعلى ذروته، ثم أيجاد قدرة التحليل للعدسات (Mulvey , 1982 ) من المعادلة التالية: $\delta=0.71\left(\mathrm{C}_{\mathrm{s}} \lambda^{3}\right)^{1 / 4}$ .. in (nm)

حيث $\lambda$ الطول الموجي المرافق لفولتية التتغيل بوحدة النانومتر ويحتسب من العلاقة التالية: $\lambda=\left(1.5 / \mathrm{V}_{\mathrm{r}}\right)^{1 / 2}$ .. in (nm)

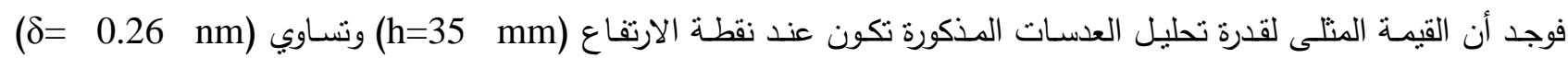
والموضحة في الثكل (10d). كما إن الثكل (10) يبين أن التخصر المقترح يجعل الخواص البؤرية للعدسة المصممة قريبة جدا لتحقيق هدف البحث في تصميم العدسة وتصنيعها وفق الثروط التطبيقية والمختبرية والمحددة بالقيم C .$(0.2 \mathrm{~nm}$

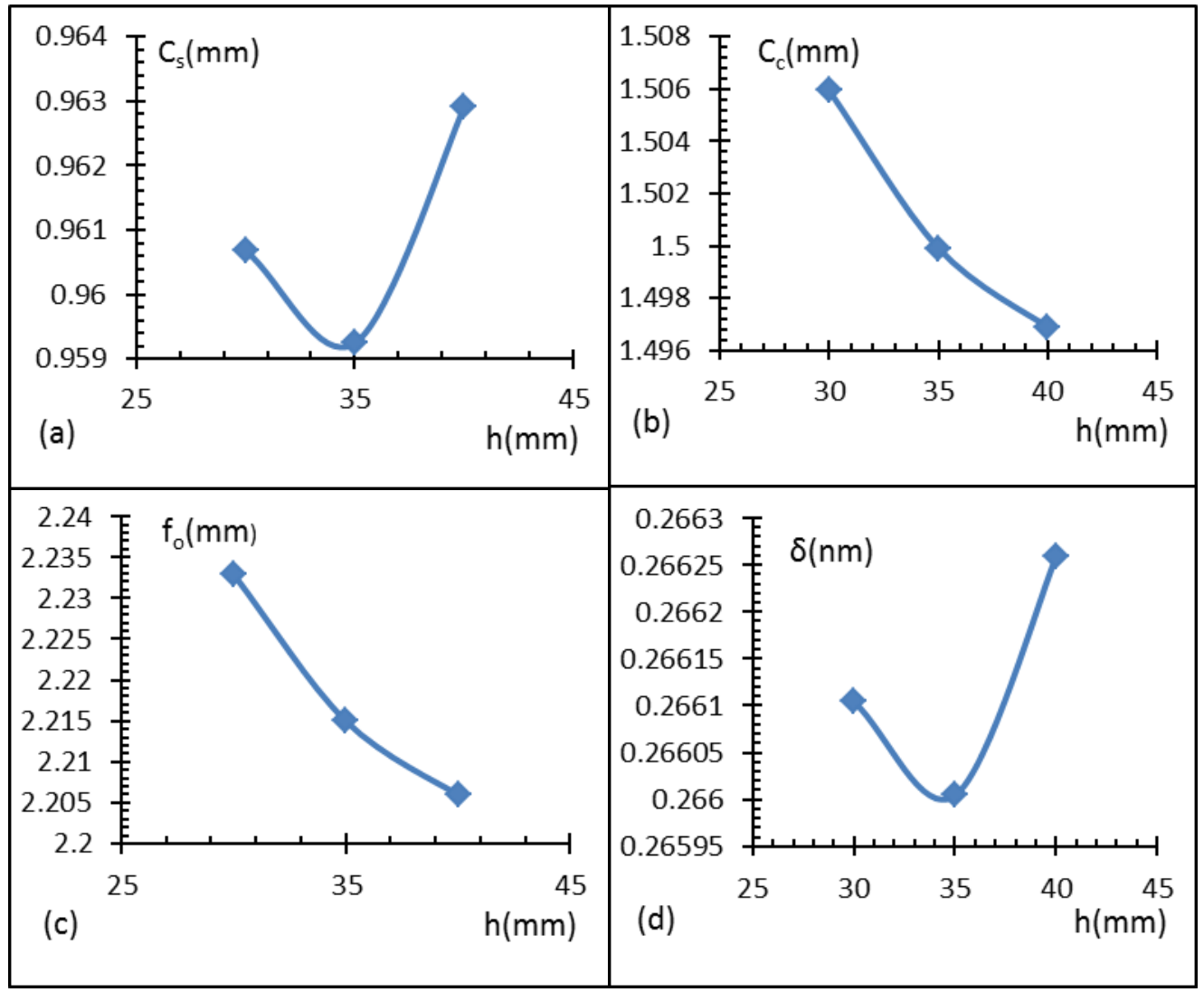

الثكل 10: تغير الخواص البصرية البؤريـة (a)- معامل الزيخ الكروي، b- معامل الزيـغ اللوني ، c- معامل

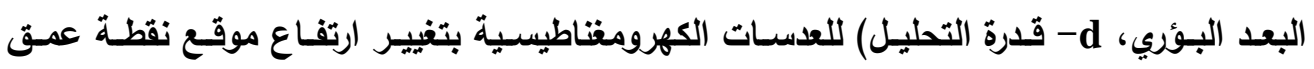
التخصر في ذراعها الحديدي عند التهيج الثابت (NI=10.5 kA-t).

ومما تجدر الإثارة إليها أن العدسة ذات الخواص المتوافقة مع الثروط المختبرية والتصنيعية المطلوبة ضمن هدف البحث الحالي عند مقارنتها مع نظيراتها عند نفس الثروط التشغيلية، هي التي تمتلك أقل معامل زيغ كروي عندما يكون موقع ذروة الفيض المغناطيسي للعدة الكهرومغناطيسية في منتصف الفجوة الهوائية ويكون موقع النموذج قريبا جدا منه في اتجاه القطب 


$$
\text { منى عبد الكريم الخشاب و ثامر جمعة الخالدي }
$$

الأول (القطب الرئيسي). بذلك يتم تحديد الثكل الأمثل للذراع الحديدي عندما تكون الخواص البصرية في أفضل قيمتها وتكون

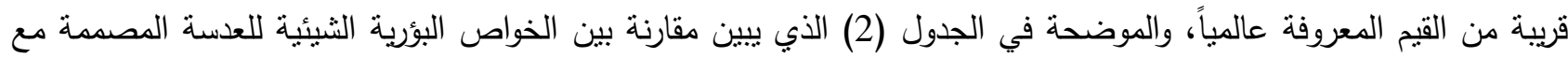

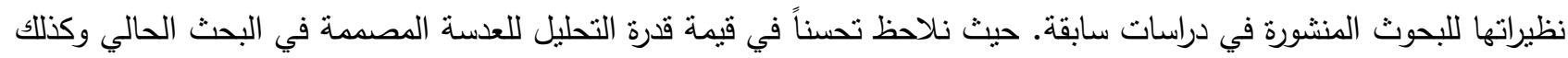

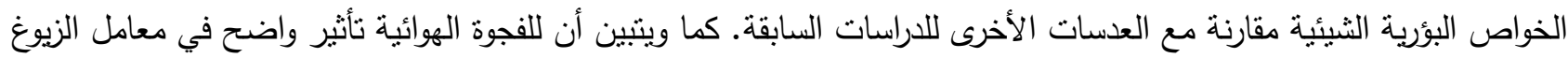
الكروي والبعد البؤري للعدسة عند تهيج ثابت وفولنية تعجيل محددة.

الجدول 2: مقارنة الذواص البصرية المثلى للعدسة الكهرومغناطيسية المصممة في البحث الجاري عند فولتية التشغيل (200 kV)

.$(\mathrm{NI})$

\begin{tabular}{|l|l|l|l|l|l|}
\hline Lens & $\mathbf{S}(\mathbf{m m})$ & $\mathbf{N I}(\mathbf{k A - t})$ & $\mathbf{C}_{\mathbf{s}}(\mathbf{m m})$ & $\mathbf{f}_{\mathbf{0}}(\mathbf{m m})$ & $\mathbf{\delta}(\mathbf{n m})$ \\
\hline Present work & 4 & 10.5 & 0.95 & 2.2 & 0.26 \\
\hline Li et al. ، 2009 & 6 & 9.976 & 1.38 & 1.8 & 0.29 \\
\hline Tsuno and Harada، 1983 & 3 & 10.9 & 0.83 & 2.4 & 0.25 \\
\hline
\end{tabular}

\section{الاستتنتاجات}

إن لسمك الذراع الحديدي أهمية كبيرة في تحديد الخواص البصرية للعدسة الكهرومغناطيسية لذا يتوجب على المصمم الاهتمام بتحديدها حسب الغرض المطلوب من تصميم العدسة، إذ أن معامل البعد البؤري للعدسة المطلوبة يتتاسب تتاسباً عكسياً مع مقدار سمك الذراع الحديدي للعدسة.

عند وضع تخصر في الذراع الحديدي للعدسة الكهرومغناطيسية نلاحظ تحسناً طفيفاً لقدرة التحليل للعدسة مع زيادة عمق التخصر إلى حد معين، مبيناً أن هناك قيمة فضلى لسمك الذراع الحديدي لكل قيمة من قيم الخواص البصرية المطلوبة، وان مقدار عمق التخصر وكذللك ارتفاعه (موقع بعده عن المحور البصري) ذو نأثير طفيف في الخواص البصرية للعدسة لكنه عامل مهم في تحيد الثنكل الهندي للذراع الحديدي وبالتالي في تحديد الثنكل الهندسي للعدسة الكهرومغناطيسية، سَيََّما عند دراسة مسارات خطوط الفيض المغناطيسي في تركيب العدسة لما لها من دور في تحديد عيوب العدة المصممة لمعرفة مدى تسرب الفيض المغناطيسي الناتج عن تمغنط الأقطاب الحديدية فيها بسبب التهيج العالي للعدسة، وتأثير هذا التسرب المغناطيسي على مسارات الحزمة الاكترونية. وبالتالي وجد أن هنالك حالة مثلى للثكل الهندسي للذراع الحديدي والتي توفر الخواص المطلوبة لتحقيق الغرض التطبيقي من العدسة المصممة.

\section{المصادر}

Al-Khashab, M.A.; Abbas, I.K. (1991). Optimized polepiece shape for single polepiece magnetic electron lenses, Modelling, Simulation and Control, A.AMSE Press, 35(3), 1-9.

Al-Khashab, M.A.; Ahmad, A.A. (2011). The effect of magnetic lens structure insulation on its optical performance. Dirasat Pure Science, 38(2), 161-168.

Al-Khashab, M.A.; Al-Hialey, R.W. (2013). The effect of the geometrical shape and position of the coil on the optical performance for asymmetrical magnetic lens. Raf. J. Sci., 24(2), 100-109.

Bleeker, A.J.; Kruit, P. (1990). Condenser objective lens with asymmetric polepieces to facilitate the extraction of secondary and Auger electron. Rev. Sci. In strum. 61(12), 350-356. 
Cleaver, J.A.R. (1980). The choice of polepiece shape and lens operating mode for magnetic objective lenses with saturated polepiece. Optik, 57, 9-34.

Krivanek, O.L.; Corbin, G.J.; DEllby, N.; Elston, B.F.; Keyse, R.J.; Murfit, M.F.; Own, C.S.; Szilagyi, Z.S.; Woodruff, J.W. (2008). An electron microscope for the aberration -corrected era. Ultramicroscopy, 108(3), 179-195.

Lencova', B. (1986). Program AMAG for computation of vector potential in rotationally symmetric electron lenses by the finite element method. Inst. Sci. Instru., Czech. Acad. Brno, (Czech),1-58.

Lenz, F. (1982). "Properties of Electron Lenses, in Magnetic Electron lenses". ed. P.W. Hawkes, Springer -Verlary, Bertin, pp.119-161.

Li, Wen-ping; Wu, Jain; Zhou Zhen; Gui, Li-Jaing; Han, Li. (2009). Practical integrated design of a condenser-objective lens for Transmission Electron Microscope. J. Physics, Conference Series, 188(2009), 012045

Mulvey, T. (1982). "Magnetic Electron Lens Properties". ed. P.W. Hawkes, Berlin, Springer, Ch.5, pp. 359-412.

Mulvey, T. (1986). Unconventional lens design and columns. Proc. XIth Int.Cong., on Electron Microscopy, Kyoto. 239-242.

Munro, E. (1975). "A Set Of Computer Program for Calculating the Properties of Electron Lenses". Cambridge University, Eng. Dep., Report CHED/ B- ELECT/TR-45, pp.13-100

Murad, W.M. (1998). Design of iron-free magnetic electron lenses. M.Sc. Thesis, University of Mosul, Iraq (in Arabic).

Podbrdsky, J. (1986). High current density magnetic electron lenses in modern electron microscope. Scan. Electr. Microscope /III, (887-896).

Ray, F.E. (2005). "Physical Principles of Electron Microscope". University of Alberta, Canada, Springer Science + Business Media, Inc. U.S.A.,Ch.3, 57 p.

Tsuno, K.; Smith, K.C.A. (1986). Optimization of objective lens shape for high resolution electron microscopes. Proc. XIth Int. Cong, on Electron Microscopy, Kyoto. 295-296

Tsuno, K.; Harada, Y. (1983). Design procedure for a high resolution electron microscope objective lens. J. Electron Microsc., 32(4), 289-298.

Wenxiong, C. (1988). The effect of polepiece saturation on the electron optical properties of asymmetrical condenser-objective lenses. Scanning Microscope, 2(3), 1283-1292.

Yanak, T. (1986). Strongly-exited CTEM/STEM objective lens . Proc. XIth Int. Cong., on Electron Microscopy, Kyoto.243, Ed -245. 